d'azote cadre bien avec cette conception, car la molécule d'azote oppose à la dissociation en atomes une résistance beaucoup plus grande que la molécule d'oxygène. Il faudra donc utiliser un mélange surazoté si l'on veut que, lors de la formation de $\mathrm{NO}$, les atomes $\mathrm{N}$ et $\mathrm{O}$ se trouvent en nombre absolu à peu près égal, de façon à réaliser les conditions de rendement optimum.

Genève, Laboratoire de Chimie technique et théorique de l'Université, Avril 1919.

Un mémoire détaillé sur le sujet de cette note paraìtra prochainement dans le Journal de Chimie Physique.

\title{
Über die Beziehungen zwischen den Jononen und Iron
}

von

L. Ruzieka.

(5. V. 19)

Tiemann und Krüger ${ }^{1}$ ) gaben dem von ihnen entdeckten Riechstoff der Iriswurzel Iron die Formel I, wonach sich derselbe von den synthetisch erhaltenen $\alpha$-Jonon und $\beta$-Jonon (II und III) nur durch die Lage der Ringdoppelbindung unterscheiden sollte.

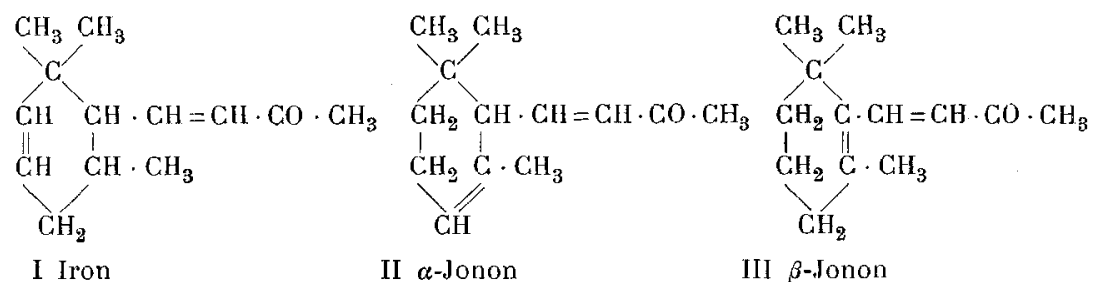

Diese Formulierung des Irons beruht hauptsächlich auf den Abbauresultaten des daraus beim Kochen mit starker Jodwasserstoffsäure und Phosphor entstehenden Kohlenwasserstoffs Iren, und dann auch indirekt (infolge des ähnlichen Geruchs) ${ }^{2}$ ) auf den
1) B. 26, 2675 (1893).
2) B. 26, 2706 (1893). 
Formeln der Jonone ${ }^{1}$, die durch direkte Oxydation mit Kaliumpermanganat sichergestellt sind ${ }^{2}$ ). Bei der Übertragung der letzteren Arbeitsweise auf Iron konnten Tiemann und Krüger ${ }^{3}$ ) nur Produkte eines weitgehenden Abbaus isolieren, aus denen kein Schluss auf die Konstitution möglich war; die ungünstige Lage der Ringdoppelbindung des Irons lässt derartige Versuche schon von vornherein wenig aussichtsreich erscheinen. Auch bei der Ozonisation besteht aus dem gleichen Grunde die Möglichkeit der Bildung eines Gemisches von Polyaldehyden und -carbonsäuren; unter Anwendung dieser Methode erhielt ich aus Iron keine charakteristischen Spaltstücke.

Da also danach der einzigen direkten Stütze der Ironformel eine Reaktion zugrunde liegt, bei der Umlagerungen möglich sind, so wären weitere Untersuchungen des Irons für die Klarlegung seiner Konstitution sehr erwünscht. $\mathrm{Zu}$ diesem Zwecke unternahm ich die katalytische Reduktion des Irons mit Platinmetallen bei gewöhnlicher Temperatur, wobei als gut charakterisiertes Produkt das Tetrahydroiron (IV) erhalten werden konnte.

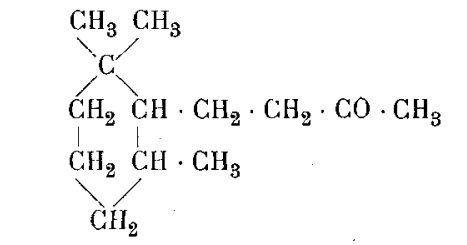

IV Tetrahydroiron (Tetrahydrojonon)

Dadurch ist auch der exakte chemische ${ }^{4}$ ) Beweis geliefert, dass das Iron wirklich zwei Doppelbindungen besitzt, denn der Nachweis derselben über das Iren ist insofern unsicher, als sich beim Kochen mit Jodwasserstoffsäure leicht Doppelbindungen aus einem bicyclischen System bilden können, wie z. B. beim Übergange vom Thujon ins Isothujon und ähnlichen Reaktionen.

Das Tetrahydroiron war noch aus einem anderen Grunde von Interesse. Nach den Formeln für die Jonone und für Iron sollte man erwarten, dass die Tetrahydroderivate dieser Körper

1) Die Syuthese der Jonone aus Citral durch stark saure Reagentien ist für die Konstitution weniger entscheidend.

2) Tiemann, B. 31, 857, 872 (1898).

3) B. 26, 2683 (1893).

4) Bisher nur durch die Molekularrefraktion. 
identisch seien bis auf die optische Aktivität: die Jonone sind bekanntlich optisch inaktiv, das Iron besitzt zwei asymmetrische Kohlenstoffatome und ist auch rechtsdrehend. Skita ${ }^{1}$ ) gibt nun an, dass die beiden Jonone dasselbe Tetrahydroprodukt liefern, ohne jedoch ein krystallisiertes Derivat zum Vergleich beschrieben zu haben. Die aus diesem Grunde hergestellten Semicarbazone des $\alpha$ - und des $\beta$-Tetrahydrojonons sind in der Tat identisch. Dagegen unterscheidet sich das Tetrahydroiron ausser durch seine Rechtsdrehung noch in anderen Eigenschaften vom Tetrahydrojonon: schon sein spezifisches Gewicht ist höher, und, was besonders auffällt, der Siedepunkt des Tetrahydroirons (ca. $143^{\circ}$, $13 \mathrm{~mm}$ ) liegt um etwa $14^{\circ}$ höher als der des Tetrahydrojonons $\left.\left(\text { ca. } 129^{\circ}, 13 \mathrm{~mm}\right)^{2}\right)$. Da die optische Isomerie keinen Siedepunktsunterschied bedingt, so kann ein solcher bei Annahme der gleichen Strukturformel nur auf Cis-trans-Isomerie beruhen. Es wurden bisher bei cis-trans-isomeren cyclischen Verbindungen in zahlreichen Fällen Siedepunktsunterschiede ${ }^{3}$ ) beobachtet, die aber nie $10^{\circ}$ übersteigen und sich gewöhnlich zwischen ca. 4-8 ${ }^{\circ}$ bewegen. Die ausnahmsweise hohe Differenz in unserem Falle dürfte vielleicht durch die relativ lange Seitenkette der Veilchenketone bedingt sein; man besitzt in dieser Beziehung allerdings mangels vergleichbarer Beispiele keinen sicheren Anhaltspunkt.

Zur Entscheidung der Strukturidentität des Tetrahydrojonons und Tetrahydroirons stehen nun zwei Wege offen: Versuche zur gegenseitigen Umwandlung oder Abbau zu gleichen Spaltprodukten. Der erstere Weg blieb sowohl beim Erhitzen mit Salzsäure wie mit Alkali auf höhere Temperatur erfolglos; das Tetrahydroiron wurde so nicht einmal racemisiert. Auch die Abbauversuche durch Oxydation sind in dieser Hinsicht gescheitert. Die gesättigten Veilchenketone sind gegen Permanganat so beständig, dass daneben die zu erwartenden empfindlicheren. Oxydationsprodukte $^{4}$ ) (Geronsäure und 1,1-Dimethyladipinsäure) nicht isolierbar waren. Auch die Oxydationsversuche mit den wässerigen

1) B. 45,3314 (1912).

2) Nach skita, 1. c. $126-127^{\circ}(13 \mathrm{~mm})$, wohl nicht korrigiert. Obige Siedepunkte beziehen sich auf ein abgekürztes Normalthermometer.

3) Vergl. die Zusammenstellung bei A.Werner, Lehrbuch der Stereochemie S. 119 und 161 fif. (1904).

4) Vergl. darüber Tiemann, B. 31, 853 (1898) und B. 33, 3724 (1900). 
Lösungen der Natriumsalze der aus den Ketonen durch Natriumhypobromit gewonnenen Tetrahydrojononsäure und Tetrahydroironsäure (V) zeigten das gleiche Ergebnis. Das Auftreten grösserer Mengen Oxalsäure in allen diesen Fällen deutet darauf hin,
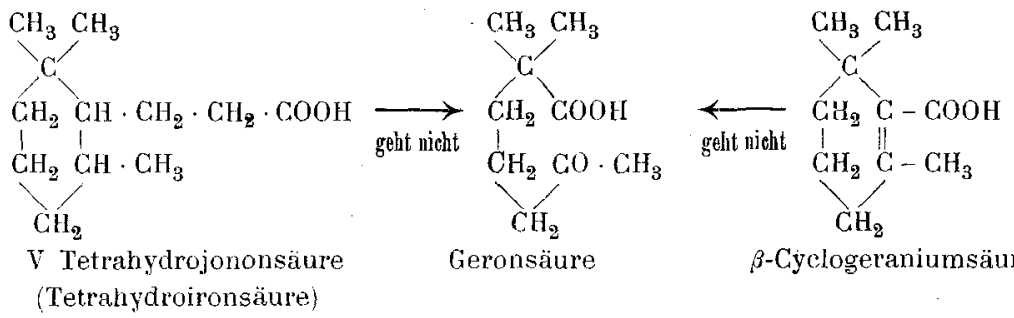

Geronsäure

$\beta$-Cyclogeraniumsäure

dass zuerst wohl die lange Seitenkette wegoxydiert wird. Der weitere Abbau verläuft dann vermutlich analog wie bei $\operatorname{der} \beta$ Cyclogeraniumsäure, aus der Tiemann und Schmidt ${ }^{1}$ ) auch die primären Oxydationsprodukte nicht fassen konnten, obwohl die Bedingungen hier infolge des Vorhandenseins einer Doppelbindung noch günstiger liegen als bei den oben genannten gesättigten Säuren.

Es würde nahe liegen, einen systematischen Abbau der Tetrahydroketone resp. der Säuren zu versuchen, wozu verschiedene Methoden in Betracht kämen; da aber letzten Endes alle solchen Versuche auf eine Abspaltungsreaktion nach einem Schema hinauslaufen müssten,

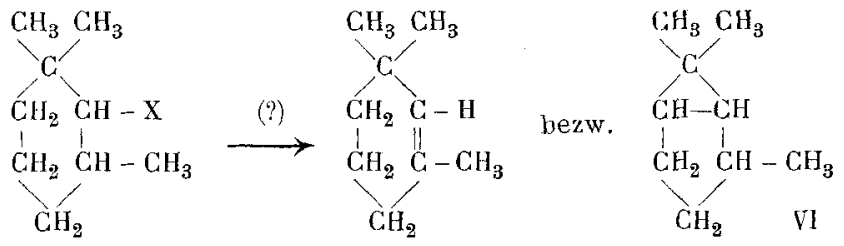

das ein klassisches Beispiel für die Möglichkeit einer Wagnerschen Umlagerung (VI) bietet ${ }^{2}$ ), so ist dieser Weg für eine Konstitutionsaufklärung zu unsicher.

Über das Tetrahydroiron die Konstitutionserforschung des Irons weiter zu verfolgen, hat daher wenig Aussicht auf Erfolg. Am

1) B. 33, $3724(1900)$.

2) In analogen Fällen sind von Meerwein, A. 405, 103 ff (1914) bei der A.bspaltungsreaktion tatsächlich nur Umlagerungsprodukte isoliert worden. Vergl. auch Helv. 1, 132 (1918). 
zweckmässigsten würde es noch sein, die Ozonisation eventuell unter Verbindung mit anderen Oxydationsmethoden an Hand grösserer Mengen Irons zu untersuchen, die aber zurzeit nicht zu beschaffen sind.

Versucht man nun auf Grund des bisher gewonnenen ${ }^{1}$ ) Materials, unter Annahme der immerhin wahrscheinlichen Ironformel von Tiemann und Krüger, sich eine Vorstellung über die Unterschiede zwischen den Jononen und Iron zu machen und berücksichtigt hierbei die Bildungsreaktion des $\beta$-Jonons, bei der eine Wasserabspaltung stattfindet, so kommt für die Jonone und das

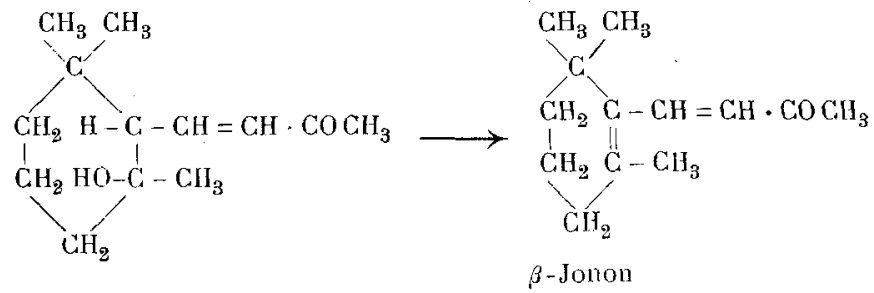

Tetrahydrojonon in erster Linie die Cis-Stellung der beiden Seitenketten an den asymmetrischen Kohlenstoffatomen in Betracht, für das Iron und Tetrahydroiron dagegen die Trans-Stellung:

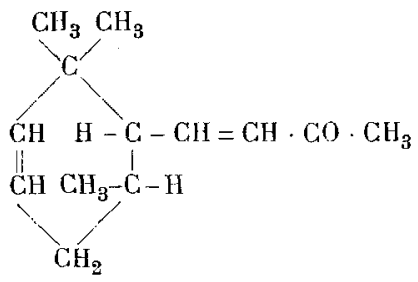

Iron

1) Lin Fünfring z. B. kommt für Iron schon deshalb nicht in Betracht, da alle lronderivate höher sieden als die der Jonone, und sonst allgemein Fünfringe einen tieferen Siedepunkt aufweisen als analoge Sechsringe. Bei einem Siebenring müssten sich wieder leichter Oxydationsprodukte fassen lassen. In diesem Zusammenhange wäre es auch von Interesse, die Konstanten des von Merling und Welde [A. 366, 199 ff (1909)] gewonnenen synthetischen racemischen Irons kennen zu lernen. Merling und Welde haben die diesbezüglichen experimentellen Daten dem schon vor zehn Jahren erschienenen theoretischen Teil ihrer schönen Untersuchungen noch nicht folgen lassen, so dass es vorläufig dahingesteilt bleiben muss, wieweit das synthetische Iron init dem natürlichea übereinstimmt. 


\section{- $357-$ \\ Experimenteller Teil. \\ Reduktion des Irons.}

Das zu den Versuchen angewandte Iron wie auch die Jonone waren von der Firma Haarmann \& Reimer, Holzminden, bezogen. Es zeigte den Sdp. von $90-95^{\circ}$ bei $0,2 \mathrm{~mm}\left(140-145^{\circ}\right.$ bei $12 \mathrm{~mm}$ ) sowie $[\alpha]_{\mathrm{D}}=+33,3^{\circ}$ in ca. $20 \%$ iger ätherischer Lösung.

a. Mit kolloidalem Palladium. $5 \mathrm{gr}$ Iron wurden in wässrig alkoholischer Lösung nach dem Verfahren von Skita mit kolloidalem Palladium und Wasserstoff geschüttelt beim Druck einer Wassersäule von etwa $30 \mathrm{~cm}$. Die erste Molekel Wasserstoff wurde in ca. 6 Stunden aufgenommen, eine weitere Molekel in zwei Tagen. Nach noch längerem Schütteln wird kein Wasserstoff mehr absorbiert. Der Siedepunkt des entstandenen Tetrahydroirons liegt bei $143-145^{\circ}(13 \mathrm{~mm})$; es ist ein farbloses Öl von charakteristischem Gederngeruch und entfärbt Brom in Schwefelkohlenstofflösung ziemlich rasch unter Bromwasserstoffentwicklung.

$[\alpha]_{\mathrm{D}}=+25,2^{\circ}$ (in ca. $6 \%$ iger ätherischer Lösung)

$\mathrm{d}_{15}=0,9274$

$0,0967 \mathrm{gr}$ Subst. gaben $0,2806 \mathrm{gr} \mathrm{CO}_{2}$ und $0,1059 \mathrm{gr} \mathrm{H}_{2} \mathrm{O}$

0,1081 gr Subst. gaben 0,3139 gr $\mathrm{CO}_{2}$ und 0,1181 gr $\mathrm{H}_{2} \mathrm{O}$

$$
\begin{array}{lll}
\mathrm{C}_{13} \mathrm{H}_{24} \mathrm{O} \text { (IV) } & \text { Ber. C } 79,58 & \text { H 12,25\% } \\
& \text { Gef. "79,18,79,21 }, 12,25,12,23 \%
\end{array}
$$

Das Semicarbazon fällt fast momentan aus und schmilzt nach dem Umkrystallisieren aus Alkohol bei 202-203 ${ }^{\circ}$.

$$
\begin{aligned}
& 0,1101 \text { gr Subst. gaben } 16,3 \mathrm{~cm}^{3} \mathrm{~N}_{2}\left(15,5^{\circ}, 715 \mathrm{~mm}\right) \\
& \mathrm{C}_{14} \mathrm{H}_{\mathbf{2 7}} \mathrm{ON}_{\mathbf{3}} \text {. Ber. N } 16,60 \% \\
& \text { Gef. "16,47\% }
\end{aligned}
$$

Bei der Spaltung des Semicarbazons durch Kochen mit Kaliumbisulfatlösung wurde das Keton regeneriert; dasselbe besass den unveränderten Siedepunkt.

$$
\begin{aligned}
& 0,0863 \text { gr Subst. gaben } 0,2522 \text { gr } \mathrm{CO}_{2} \text { und } 0,0943 \text { gr } \mathrm{H}_{2} \mathrm{O} \\
& \mathrm{C}_{13} \mathrm{H}_{24} \mathrm{O} \text { (IV) Ber. C 79,58 H 12,25\% } \\
& \text { Gef. „ } 79,73 \quad \text { "12,23\% }
\end{aligned}
$$

b. Mit Platinschwarz. Auch bei der Reduktion nach der Methode von Willstätter mit 1 gr Platinschwarz in ätherischer 
Lösung wurden von $5 \mathrm{gr}$ Iron in etwa 10 Stunden 2 Mol. Wasserstoff aufgenommen. Bricht man nun die Reduktion ab, so erhält man, nach dem Siedepunkt des Ketons und dem Schmelzpunkt des Semicarbazons zu schliessen, das gleiche Tetrahydroiron.

\section{Versuche zur Darstellung des Dihydroirons.}

Lässt man etwas weniger als 1 Mol. Wasserstoff in Gegenwart von kolloidalem Palladium auf Iron einwirken, so dass noch unveränderte Substanz in der Lösung vorhanden sein muss, und stellt daraus das Semicarbazon her, so zeigt sich die Anwesenheit von Iron durch die entstandenen etwas klebrigen Krystalle an, da das Iron nur ein amorphes, schwer krystallisierendes Semicarbazon liefert. Nach dem Umkrystallisieren aus Alkohol und Entfernen des leicht löslichen Ironsemicarbazons lässt sich kein einheitliches Semicarbazon gewinnen. Es besitzt auch frach oftmaligem Umkrystallisieren je nach den einzelnen Fraktionen einen sehr unseharfen Schmelzpunkt zwischen 170-200'.

Lässt man etwas mehr als 1 Mol. Wasserstoff auf Iron einwirken, so kann man durch mehrmaliges Umkrystallisieren des Semicarbazons reines Tetrahydroironsemicarbazon von Schmelzpunkt und Mischprobe $202-203^{\circ}$ erhalten. Das mit $1 \mathrm{Mol}$. Wasserstoff behandelte Iron enthält also schon beträchtliche Mengen Tetrahydroiron. Es ist daher auch unsicher, ob Skita ${ }^{1}$ ) bei der analogen Behandlung der Jonone die reinen Dihydrojonone in den Händen gehabt hat, da er keine krystallisierten Derivate beschrieb. Die Ringdoppelbindung des Irons scheint wenigstens teilweise noch vor der der Ketogruppe benachbarten Kohlenstoffdoppelbindung reduziert zu werden.

\section{Reduktion der Jonone.}

Nach $\left.S k i t a^{2}\right)$ absorbieren die beiden Jonone beim Schütteln mit kolloidalem Palladium in wässrig alkoholischer Lösung bei $1 \mathrm{Atm}$. Ueberdruck in etwa einer Stunde 2 Mol. Wasserstor und liefern ein Tetrahydroprodukt vom gleichen Sdp. $126-127^{\circ}(13 \mathrm{~mm})$. In essigsaurer Lösung 3$)$ wird durch denselben Katalysator (bei hohem Ueberdruck) in etwa vier Stunden unter Aufnahme von 3 Mol. Wasserstoff der Alkohol gebildet, den man statt Hexahydrojonon zweckmässiger als T'etrabydrojonol bezeichnen kann.
1) B. $45,3313(1912)$.
2) B. 45, 3314 (1912).

3) B. 48, 1490 und 1495 (1915). 
a. Mit kolloidalem Palladium. Bei gewöhnlichem Druck verläuft die Wasserstoffaufrahme nach meinen Versuchen wesentlich langsamer. So waren z. B. für die Reduktion von $28 \mathrm{gr} \beta$ Jonon (Sdp. $135-140^{\circ}, 16 \mathrm{~mm}$ ) zu Tetrahydrojonon nach dem Verfahren von Skita einige Tage nötig. Das erhaltene Reduktionsprodukt siedet bei $131-134^{\circ}(16 \mathrm{~mm})$ und liefert mit alkoholischer Semicarbazidacetatlösung innerhalb einiger Minuten quantitativ ein Semicarbazon, das nach dem Umkrystallisieren aus Alkohol bei $183-184^{\circ}$ sehmilzt.

$0,1220 \mathrm{gr}$ Subst. gaben $0,2964 \mathrm{gr} \mathrm{CO}_{2}$ und $0,1175 \mathrm{gr} \mathrm{H}_{2} \mathrm{O}$

$$
\begin{aligned}
& \mathrm{C}_{14} \mathrm{H}_{27} \mathrm{ON}_{3} \text { B } 68 \text {. C } 66,40 \text { H } 10,68 \% \\
& \text { Gef. "66,28 "10,76\% }
\end{aligned}
$$

Ein in der gleichen Weise aus $\alpha$-Jonon (Sdp. 128-130", $10 \mathrm{~mm}$ ) hergestelltes Tetrahydrojonon siedete bei $128-130^{\circ}(14 \mathrm{~mm})$ und liefert ein nach Schmelzpunkt und Mischprobe mit obigem identisches Semicarbazon. Von zwei verschiedenen Fraktionen des letzteren Tetrahydrojonons wurde das spez. Gew. zu $d_{15}=$ 0,9158 und 0,9151 gefunden.

b. Mit Platinschwarz. Bei der Reduktion nach der Methode von Willstätter in ätherischer Lösung mit $1 / 5$ des Gewichtes Platinschwarz unter Atmosphärendruck werden in etwa 8 Stunden 2 Mol. Wasserstoff aufgenommen und wenn man jetzt aufarbeitet, kann aus dem Öl das gleiche Semicarbazon erhalten werden. Schïttelt man jedoch weiter, so wird noch 1 Mol. Wasserstoff in etwa zwei Tagen absorbiert und es entsteht das Tetrahydrojonol (Alkohol entsprechend Formel IV) vom Sdp. 142-143 ${ }^{\circ}(17 \mathrm{~mm})$. Es ist dickflüssiger als das Keton, liefert kein Semicarbazon und zeigt schwachen Cederngeruch von einer angenehmeren Nuance als der des Ketons.

$$
\left.\mathrm{d}_{15}=0,9144^{1}\right)
$$

0,0832 gr Subst. gaben 0,2400 gr $\mathrm{CO}_{2}$ und 0,0974 gr. $\mathrm{H}_{2} \mathrm{O}$

$$
\begin{aligned}
\mathrm{C}_{12} \mathrm{H}_{26} \mathrm{O} & \text { Ber. C } 78,77 \text { H 13,13\% } \\
& \text { Gef. } 78.70 \quad \# 13,10 \%
\end{aligned}
$$

Auch aus diesem Beispiele erhellt die wiederholt beobachtete energischere Reduktionswirkung von Platinschwarz im Vergleich zum kolloidalen Palladium, das nach den Angaben Skitas in wässrig alkoholischer Lösung sogar bei 1 Atm. Überđruck Tetrahydrojonon nicht mehr weiter reduziert.

1) Nach Skita, B. 48, 1490 und 1495 (1915), ist der Sdp. 142-1430 $(20 \mathrm{~mm})$ und $\mathrm{d}_{20}=0,9126$. 
c. Mit Nickel. Wesentlich verschieden verläuft die Reduktion mit dem Nickelkatalysator von Kelber ${ }^{1}$ ). $25 \mathrm{gr} \alpha$-Jonon wurden in einer Lösung von $50 \%$ igem Alkohol einige Wochen mit Wasserstoff geschüttelt unter oftmaligem Zusatz von je $10 \mathrm{gr}$ des 20\%igen Katalysators (auf Klärerde niedergeschlagen). Die Wasserstoffaufnahme verlief sehr langsam und war nur kurze Zeit nach dem erneuten Katalysatorzusatz einigermassen von Bedeutung. Die Menge des aufgenommenen Wasserstoffs konnte so nicht genau bestimmt werden. Das derart behandelte öl zeigte den Sdp. $135-140^{\circ}(14 \mathrm{~mm})$, ist dickflüssiger als das Tetrahydrojonon und lieferte keine Spur Semicarbazon. Es wurde danach anfangs für Tetrahydrojonol angesehen und sollte durch Oxydation mit der berechneten Menge Chromsäure in Eisessiglösung auf dem Wasserbade ins Keton übergeführt werden. Das Oxydationsprodukt zeigt fast quantitative Semicarbazonhildung; letzteres wurde durch Destillation mit Wasserdampf mit Kaliumbisulfatlösung gespalten. Das erhaltene Keton ist dünnflüssig, im Geruch verschieden vom Tetrahydrojonon und siedet bei $130-132^{\circ}$ (14 mm).

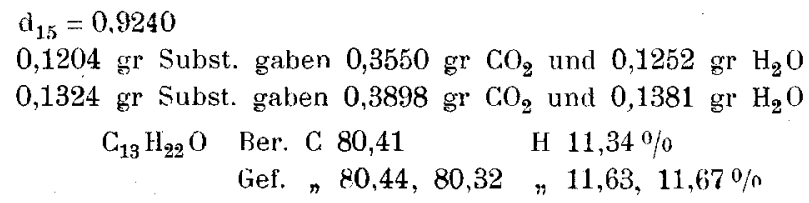

Das daraus quantitativ entstehende Semicarbazon schmilzt nach dem Umkrystallisieren aus Alkohol bei $166-168^{\circ}$ und zeigt die Zusammensetzung des Dihydrojononsemicarbazons.

0,1061 gr Subst. gaben $0,2590 \mathrm{gr} \mathrm{CO}_{2}$ und $0,0972 \mathrm{gr}_{2} \mathrm{O}$

$$
\begin{aligned}
& \mathrm{C}_{14} \mathrm{H}_{25} \mathrm{ON}_{3} \text { Ber. C } 66,92 \text { H } 9,96 \% \\
& \text { Gef. ,66,61 \#10,25\% }
\end{aligned}
$$

Es liegt hier wohl ein ziemlich reines Dihydrojonon, $\mathrm{C}_{13} \mathrm{H}_{22} \mathrm{O}$, vor und der ursprünglich bei der Reduktion entstandene Alkohol wäre als Dihydrojonol aufzufassen. Der Nickelkatalysator scheint also die Reduktion der Carbonylgruppe vor der einen Kohlenstoffdoppelbindung im $\alpha$-Jonon (wahrscheinlich der im Ringe) zu begünstigen. Ein ähnlicher Fall ist meines Wissens bei katalytischen Reduktionen bisher noch nicht

1) B. 49, 55, 1868 (1916). 
beobachtet worden. Dass der Nickelkatalysator auch sonst Carbonylgruppen leicht angreift, wurde kürzlich von Rupe, Werder und Takagi ${ }^{1}$ ) beim Camphoryliden-3-aceton gezeigt.

d. Mit Zink und Natronlauge. Schliesslich wurde noch versucht - in der Erwartung, etwa ein isomeres Tetrahydrojonon zu gewinnen - das $\beta$-Jonon durch Zinkstaub und Natronlauge in alkoholischer Lösung auf dem Wasserbade zu reduzieren. Hiebei entstehen als Hauptprodukt Harze und nur ca. $20 \%$ des Reaktionsprodukts sieden unscharf zwischen $100-150^{\circ}(12 \mathrm{~mm}$ ), besitzen einen angenehmen charakteristischen Geruch und wurden nicht weiter untersucht.

\section{Tetrahyḋoironsäure (Formel V).}

9,5 gr Tetrahydroiron wurden mit einer Lösung von Natriumhypobromit (dargestellt aus $50 \mathrm{gr}$ Natriumhydroxyd in $500 \mathrm{~cm}^{3}$ Wasser und 50 gr Brom) in 2 Portionen je 5-6 Stunden unter Dampfheizung geschüttelt. Die eisgekühlte Lösung wurde mit überschüssigem Natriumbisulfit versetzt und mit Schwefelsäure angesäuert, das ausgeschiedene $\ddot{O} 1$ in Äther aufgenommen und bei $15 \mathrm{~mm}$ destilliert. Neben unverändertem Tetrahydroiron erhält man 5,5 gr der Säure vom Sdp. $185-190^{\circ}$; dieselbe erstarrt fast ganz (Smp. roh ca. $80^{\circ}$ ) und ist in allen Lösungsmitteln sehr leicht löslich. Mit Natronlauge bildet sie eine seifenartige Lösung, woraus das Natriumsalz durch Kochsalz gefällt werden kann.

$0,0998 \mathrm{gr}$ Subst. gaben $0,2669 \mathrm{gr} \mathrm{CO}_{2}$ und $0,1007 \mathrm{gr} \mathrm{H}_{2} \mathrm{O}$

$$
\begin{array}{lll}
\mathrm{C}_{12} \mathrm{H}_{22} \mathrm{O}_{2} & \text { Ber. C } 72,73 & \text { H } \mathbf{1 1}, 11 \% \\
& \text { Gef. }, 72,98 & \\
& & \mathbf{1 1}, 28 \%
\end{array}
$$

\section{Tetrahydrojononsäure (Formel V).}

Kleinere Mengen lassen sich nach der für die Tetrahydroironsäure beschriebenen Methode herstellen. Sollen grössere Mengen auf einmal gewonnen werden, so ist zu beachten, dass das in Alkali nur schwer lösliche Natriumsalz der Tetrahydrojononsäure ausgeschieden wird und das Tetrahydrojonon vor der weiteren Einwirkung des Natriumhypobromits schïtzt. Man muss daher die Reaktion etwa alle 3 Stunden abbrechen und nach dem Abheben des Öls das darin suspendierte Natriumsalz der Tetrahydrojononsäure durch Schütteln mit Wasser entfernen. Das unangegriffene Keton kann mit derselben Hypobromitlösung weiter geschüttelt werden. Es wurden so aus $25 \mathrm{gr}$ Tetrahydrojonon

1) Helv. 1, 337 (1918). 
durch dreimalige Wiederholung der Operation neben $10 \mathrm{gr}$ unveränderten Ketons $10 \mathrm{gr}$ destillierte Säure erhalten, die ein zähes Öl vom Sdp. $173-178^{\circ}(12 \mathrm{~mm})$ darstellt.

$$
\begin{aligned}
& 0,1440 \text { gr Subst. gaben } 0,3820 \mathrm{gr} \mathrm{CO}_{2} \text { und } 0,1426 \mathrm{gr} \mathrm{H}_{2} \mathrm{O} \\
& 0,1174 \text { gr Subst. gaben } 0,3115 \mathrm{gr}^{\mathrm{g}} \mathrm{CO}_{2} \text { und } 0,1180 \mathrm{gr} \mathrm{H}_{2} \mathrm{O} \\
& 0,0867 \text { gr Subst. verbrauchten } 4,42 \mathrm{~cm}^{3} 0,1 \text {-rı. Alkali } \\
& \begin{array}{llllll}
\mathrm{C}_{12} \mathrm{H}_{22} \mathrm{O}_{2} & \text { Ber. } \mathrm{C} 72,73 & \mathrm{H} 11,11 \% & \text { Aequ. Gew. } 198 \\
& \text { Gef. } 772,38,72,46 & \geqslant 11,08,11,26 \% & ; & & 197
\end{array}
\end{aligned}
$$

\section{Oxydalionsversuche.}

Tetrahydrojonon. 5,1 gr Keton wurden in wässriger Suspension $\left(200 \mathrm{~cm}^{3}\right)$ mit der einem Atom Sauerstoff entsprechenden Menge Kaliumpermanganat $(=2,8 \mathrm{gr}$ ) geschüttelt und nach der in einigen Stunden erfolgten Entfärbung mit weiteren $5,6 \mathrm{gr}$ Kaliumpermanganat wieder bis zur Entfärbung (Dauer 6 Tage) behandelt Da nach nochmaligem Zusatz von $5,6 \mathrm{gr}$ Permanganat in 6 Tagen keine Entfärbung eintrat, wurde wie üblich aufgearbeitet und dabei 4 gr unverändertes Keton zurückerhalten. Der saure Anteil besteht hauptsächlich aus Oxalsäure.

Die Oxydation des Tetrahydroirons zeigte einen analogen Verlauf.

Tetrabydrojononsäure. Lässt man die drei Atomen Sauerstoff entsprechende Menge Kaliumpermanganat auf die wässrige Lösung des Natriumsalzes der Tetrabydrojononsäure einwirken, so ist nach etwa zweitägigem Schütteln Entfärbung eingetreten und es können reichliche Mengen Oxalsäure nachgewiesen werden. Die Tetrahydrojononsäure ist noch zum grossen Teil unverändert. Es wurden deshalb andere Proben mit viel Permanganat (bis zu 14 Atom Sauerstoff) längere Zeit (bis 10 Tage) geschüttelt; auch so konnte von krystallisierten Säuren nur Oxalsäure gewormen werden. Die nebenbei entstehenden öligen und zähflüssigen Oxydationsprodukte gaben nur sehr wenig Kupfersalz und kein krystallisiertes Semicarbazon. Beim Destillieren tritt weitgehende Zersetzung ein.

Auch bei der Oxydation der Tetrahydroironsäure wurden ähnliche Ergebnisse erzielt. In einem Falle konnten hiebei noch geringe Mengen einer bei etwa $180^{\circ}$ schmelzenden Säure (Dimethylmalonsäure?) isoliert werden.

\section{Umlagerungsversuche.}

Tetrahydroiron. Beim Erhitzen des Ketons mit der fünffachen Menge $15 \%$ iger Salzsäure während 6 Stunden im Bombenrohr auf ca. $180^{\circ}$ tritt nur schwache Braunfärbung ein. Das regenerierte Produkt ist nach Siedepunkt, Schmelzpunkt und Mischprobe seines Semicarbazons, sowie Drehungsvermögen mit dem Ausgangsketon identisch. Auch nach mehrstündigem Erhitzen des Tetrahydroirons in derselben Weise auf $280^{\circ}$ wird daraus das gleiche Semicarbazon erhalten.

Für den Umlagerungsversuch durch Alkali wurde das Keton mit der gleichen Menge 50\% 
Stunden auf $150^{\circ}$ und dann kurze Zeit bis auf $220^{\circ}$ erhitzt (im Bombenrohr), ohne dass im Siedepunkt und Drehungsvermögen des noch unveränderten Teils der Substanz eine Aenderung beobachtel werden konnte; es wird nur ein grosser Teil des Ketons kondensiert.

Tetrahydrojonon bleibt bei ähnlicher Behandlung mit Salzsäure (bei $\left.180^{\circ}\right)$ und Kalilauge $\left(150^{\circ}\right)$ gleichfalls unverändert.

Kurze Zusammenfassung:

Der Unterschied zwischen den Veilchenriechstoffen $\alpha$ - und $\beta$-Jonon einerseits und Iron andererseits besteht unter Zugrundelegung der allgemein gebräuchlichen, aber noch nicht streng bewiesenen Ironformel von Tiemann und Krüger nicht nur in einer verschiedenen Lage der Ringdoppelbindung - wie bisher angenommen wurde --, sondern auch in einer Cis-trans-Isomerie, wobei für die Jonone die Cis- und für Iron die Trans-Stellung der bezüglichen Substituenten wahrscheinlich ist.

Zürich, Chemisches Institut der Eidg. Techn. Hochschule.

\section{Über optisch aktive Ketone: Ketone des 1,2,2,3-Cyclopentans}

von

H. Rupe und C. A. Kloppenburg.

(12. V. 19.)

Bis vor kurzer Zeit waren optisch aktive Ketone und Diketone der aliphatischen Reihe oder der cyclischen Verbindungen mit extracyclischer Stellung der Ketogruppen kaum bekannt ${ }^{1}$ ). Im Verlaufe unserer Untersuchungen über den Zusammenhang von optischer Aktivität mit chemischer Konstitution erwies es sich aber als notwendig, diese Liicke auszufüllen, da von der Untersuchung dieser Körper, besonders auch im Hinblick auf ihre grosse Reaktions- und Verbindungsfähigkeit, wichtige Aufschlüsse

1) Die Konstitution des Pulegon-acetones von Barbier, C. R. 127, 870 (1890) ist unsicher. Vergl. auch Winzer, A. 257, 317 (1890). 